\title{
Novel Cluster Rotating and Routing Strategy for software defined Wireless Sensor Networks
}

\author{
Dr. S. R. Mugunthan, \\ Associate Professor, \\ Department of Computer Science and Engineering, \\ Sriindu college of Engineering and Technology, \\ Sheriguda, Hyderabad, India. \\ Email: srmugunth@gmail.com
}

\begin{abstract}
The biggest problems faced by the software defined wireless sensor network are energy conservation and load balancing techniques which impose a high level of constraint. In general clustering is used in a network in order to decrease the use of energy thereby enhancing lifetime of the network. Hot spot problems are a common issue caused due to drainage of battery when there are more multi-hop networks that are close to the base station. In order to overcome this restriction, we have proposed the use of multilayer clustering architecture that is used to choose the intra and inter-cluster communication, rotation of cluster head and forwarding node. Using the routing table, the proposed methodology will be able to efficiently handle the rotation of the forwarder node. The rotation takes place based on the residual energy's threshold levels and also exploits the non-forwarder node, backup forwarder node, forwarder node and decision maker node to enhance the routing strategy of the WSN. Testing and evaluation of the proposed work is done using C programming language and results show that this methodology has better results than EADUC and TLPER as far as hop count, communication and energy consumption are taken into consideration in the cluster formation.
\end{abstract}

Keywords: forwarder node; cluster design; multi-layer; software define network; Wireless sensor network; Software define network;

\section{Introduction}

In the recent years, there is demand for new technologies that couple the use of wireless sensing, communication and other network technology in order to provide a sensor network that is application in a number of environments [1] such as natural monitoring, habituates monitoring, fire detection, enermy monitoring, healthcare, tracking, surveillance, environment monitoring etc. In general, the sensor nodes will hold a processor [2], a memory, a microcontroller [3] and a battery. Most of the applications involve the use of sensors that are unattended and are enhanced with the use of energy resources that are non-renewable, in order to perform their function for a prolonged period of time. This type of network will rely on efficient routing protocol that uses multi-hop communication [4-5] to transmit the information from source to destination. The sensor nodes are responsible for the transmission of information and tend to drain energy thereby decreasing the lifetime of the network. Hence in order to maximize network lifetime and minimize energy constraint which will require routing protocol's appropriate design [6]. The sensor nodes are located far away [7] from each other in large scale network which means direct communication proves to be difficult. In order to deal with these situations, multihop communication is the best means of routing. Irrespective of the large scale network and small scale network, transfer of data packets will require the nodes which are near the sink node which will result in battery draining [8] within a short interval. In a similar manner, excessive data transmission will take place when direct mode communication is also used. This also reduces the lifetime of the network at a fast pace. Thus WSN faces a major constraint of limited energy which prevents it from being using for a prolonged period of time. The proposed paper introduces a novel 
Journal of ISMAC (2020)

Vol.02/ No.03

Pages: $140-146$

http://irojournals.com/iroismac/

DOI: https://doi.org/10.36548/jismac.2020.3.001

methodology which uses both clustered and flat network [10]. This methodology also makes use of multi-hop and direct routing in order to improve the energy consumption efficiency. In cases of sensor and wireless ad-hoc network [9], the reassignment of forwarding nodes and rotation of cluster header is generally predefined during the design phase.

\section{Architecture}

The architecture of the proposed work is developed based on the how routing takes place, how clusters are formed and the various scenarios faced by the sensor nodes. We have taken into consideration a large set of sensor nodes in a large scale WSN [11-12]. With the help of localization technique, information on the node's location are determined. Radius of communication for individual nodes can be represented as follows:

$$
R(x, y)=\left\{A, z \in S: \mid D(A-z) \leq y_{A}\right.
$$

Where S represents the deployed nodes, $R$ represents radius of communication and $D(A-z)$ is distance between nodes $\mathrm{A}$ and $\mathrm{q}$ which are also considered to be neighbours. We have considered that the proposed work using AWGN channel which makes use of a wireless medium. The SNR is considered to be controlled in such a manner that a probability of over 0.5 .

\section{Proposed Scheme Operation}

The major issues related to throughput, routing and energy are taken into consideration which has resulted in dividing the work into three stages thereby addressing and resolving the issues. The three stages involved are routing, rotation of cluster head and selection of cluster head and forwarder node [13]. When there are a large number of nodes present in a region, the nodes can be divided into two categories namely forwarder node and listener node. Similarly the network is divided into two layers where first layer holds the details of the listener node which broadcasts information on density. This broadcasted message is received by the neighbouring nodes which set up the table holding information on node density and ID. From this table, the node with high density information is chosen and considered to be the forwarding node. This node must also be able to bear the addition of more nodes apart from the current nodes in a region with which it is connected. Using a 2-tier mechanism, the rotation of the first node is introduced. Thus in the first level, sharing of load is done using load balancing strategy and the next step involves the transfer of forwarder node from the cluster head to the next node. In a similar fashion, rotation of cluster jobs also takes place in a sequential manner. To summarize the first layer's cluster head will forward the information that they hold and in the second layer this information is further disseminated and sent to the base station.

\subsection{Rotation of cluster head}

The role of a cluster head as well as, the rotation count plays a vital role in energy squeezing. After the role of cluster head has been rotated to 'n' nodes, it is sent to an appropriate node which is said to hold high node density and residual energy thereby contributing to serving as a good means of determining the matrix, when compared 
Journal of ISMAC (2020)

Vol.02/ No.03

Pages: $140-146$

http://irojournals.com/iroismac/

DOI: https://doi.org/10.36548/jismac.2020.3.001

with other contestants. During the first iteration, each node has equal probability of being selected as the next cluster head. Hence, in order to address this issue, it is best to choose the node which has the following probability

$$
\rho_{i}=\frac{1}{\pi r^{2} \sigma}
$$

where node density is represented as $\sigma$ such that $T_{n}=T_{a}$ such that the total geographical region of the deployed node is denoted by $T_{a}=25,000$ and the total number of nodes represented by $T_{n}=600$. The major reasons for cluster head rotation are conversion to non-optimal and energy depletion. In order to address these issues a number of algorithm are used for the process. In some cases the cluster node is chosen to be a node which has memory, high residual energy and similar aspects. In that case, the proposed methodology for rotation will not disrupt the operation of the entire network. For rotation of cluster head, a threshold scheme is taken into consideration. A good example is the consideration of minimum hops. Hence the node which is closest to the base station based on the number of hops has a higher probability of being chosen. When there are more than one nodes which satisfy the equal minimum hop condition, then in order to decide the cluster head, the residual energy of the nodes can be considered. For efficient selection and process of cluster head rotation, there are two steps involved namely backup forwarding node and load balancing. The initial energy of the node is saved in a table during two criteria:

- When there is a drop in the energy level during role transfer process, to about $20 \%$ of initial energy.

- When about $50 \%$ of its initial energy is the level to which the energy dips.

When the condition of load balancing threshold is attained, the cluster head role switching is initiated in order to share the load. Load balancing threshold is expressed as follows where $E_{i}$ is the $i^{\text {th }}$ node's Energy Level and $E_{c}$ is the current node's Energy Level.

$$
E_{c}=\frac{E_{i}}{2}
$$

Here, rotation of the cluster head is messaged to the nodes of the cluster to obtain data regarding energy. This is used to decide the next cluster head taking into consideration the nodes which have higher energy data. During the selection process, till a new node is selected and the role of the cluster head is assigned, the existing cluster head will act as the backup forwarder node. When the role transfer is complete, the decision is broadcasted and communicated to all the nodes in the cluster. The nodes which have obtained acknowledgement will initiate communication with the new cluster head while the other nodes will be proceeding with their existing cluster head till they receive the acknowledgement. When the energy of the existing cluster head, reaches about $20 \%$ of its initial energy, it will send a message to its nodes stating that its role transfer is complete and the nodes begin to establish communication with the new cluster head. 
Journal of ISMAC (2020)

Vol.02/ No.03

Pages: $140-146$

http://irojournals.com/iroismac/

DOI: https://doi.org/10.36548/jismac.2020.3.001

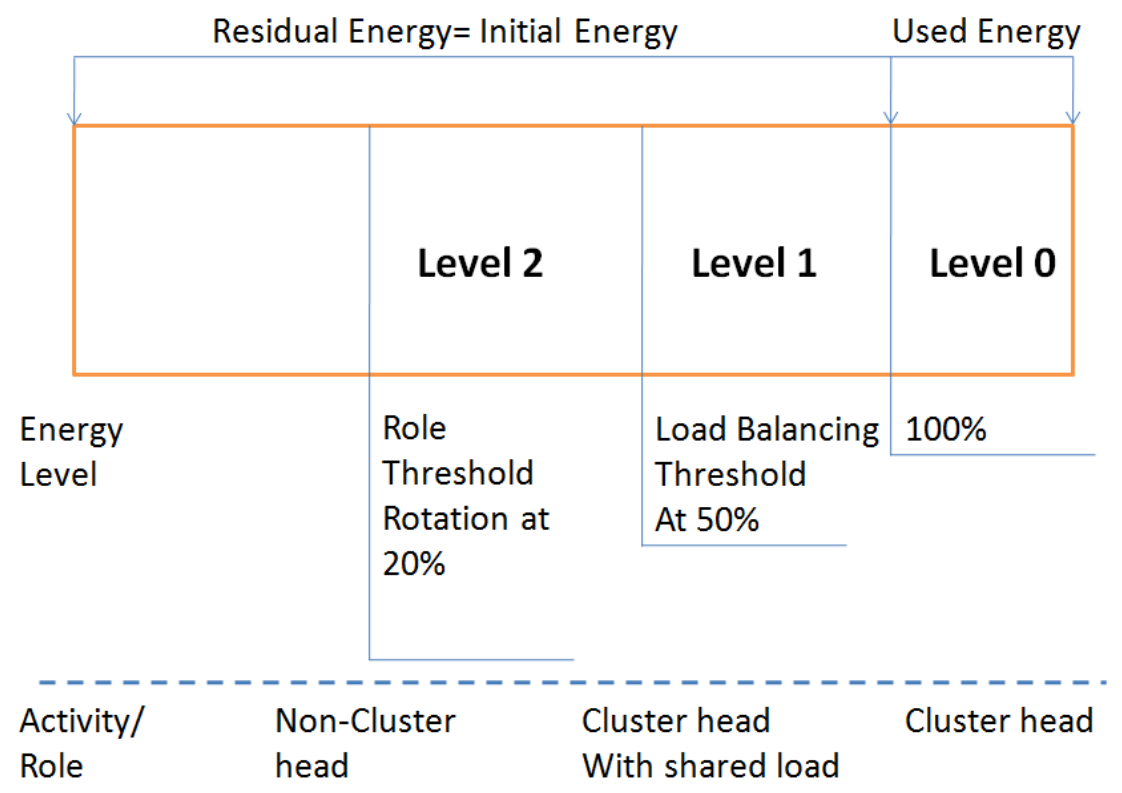

Fig.1. Role Switching of the Cluster Head with Respect to the Threshold Energy Level

\subsection{Cluster Routing Strategy}

The proposed work a designed cluster is used to rotate the role of the cluster which will aid in increasing the lifetime of the network. Moreover, node deaths are also prevented using the proposed methodology. By using balanced algorithm, it is possible to prevent WSN void problem. Using a series of steps, switching is performed as the first step.

- The decision maker node is used to determine the forwarded node based on the node which satisfies the condition $\mathrm{DM}_{\text {Node }} \rightarrow \mathrm{F}_{\text {Node }}=\mathrm{Control}_{\mathrm{x}(\mathrm{i})}>\mathrm{Control}_{\mathrm{m}(\mathrm{j}) \text { such }}$ where the letter $\mathrm{j}$ represents 1 to $\mathrm{n}$. Depending on the value the highest neighbouring node will be sent as the forwarder node.

- If the threshold is reached, a backup forwarder node is determined to aid the node. The switch between the two nodes take place when the forwarder node becomes a non-forwarder node and the backup forwarder node will be upgraded.

- At the initiation, the forwarder node role will be played by the decision maker node. It is found that after the decision making process, it takes on the role of a non-forwarder role. Similarly, all nodes which are not used in a cluster are also declared to be non-forwarder node.

- In a typical cluster, data from the nodes are sent to the cluster heads which also has a table of routing nodes with IDs of which the ID having higher ID will be chosen.

- Data is sent directly to the cluster head if the forwarder node isn't the cluster head. On receiving the data, these cluster heads will further send it to the neighbouring node which has the highest value of degree.

- This cycle will continue till data successfully reaches its destination which is the base station. 
Journal of ISMAC (2020)

Vol.02/ No.03

Pages: $140-146$

http://irojournals.com/iroismac/

DOI: https://doi.org/10.36548/jismac.2020.3.001

\section{Results and Discussion}

Fig.2 represents the rotation of cluster head. In order to determine the performance of the proposed scheme, two scheme namely EADUC and TLPER are taken into consideration depending on the routing technique used (intra and inter-routing algorithm) and are compared with the help of $\mathrm{C}$ programming. The most crucial role during the routing process is played by energy consumption which takes into account all events from initiation of cluster formation till the data is received at the base station. The Fig. 3 represents the energy consumed by the node. The node is connected to about 450 sensor nodes which are distributed within a range of $640 \mathrm{~m}$ x $640 \mathrm{~m}$. Results indicate the proposed methodology will consume lesser amount of energy when compared to the other methodologies.

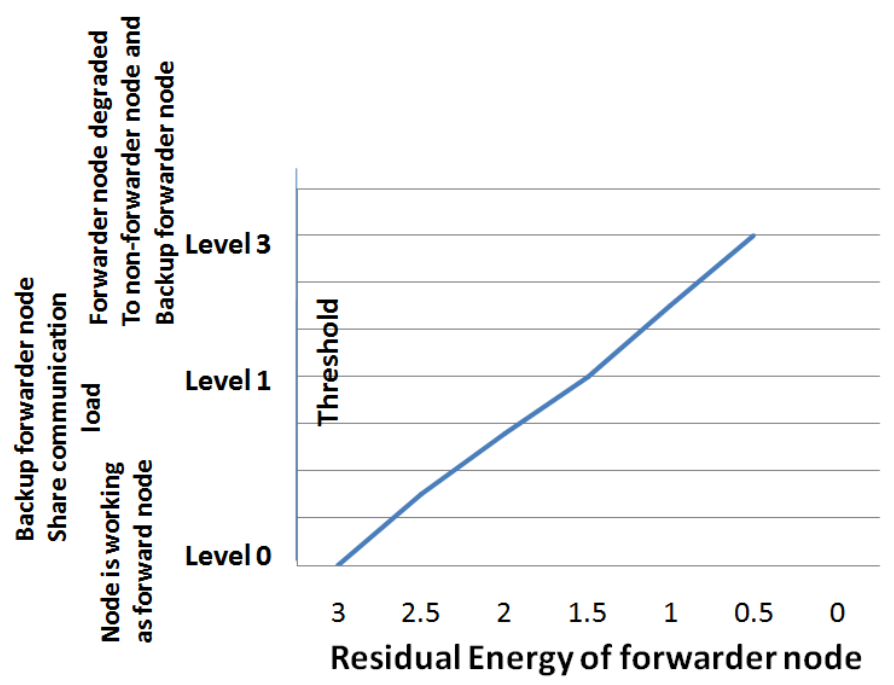

Fig.2. Residual Energy of Forwarder Node 
Journal of ISMAC (2020)

Vol.02/ No.03

Pages: $140-146$

http://irojournals.com/iroismac/

DOI: https://doi.org/10.36548/jismac.2020.3.001

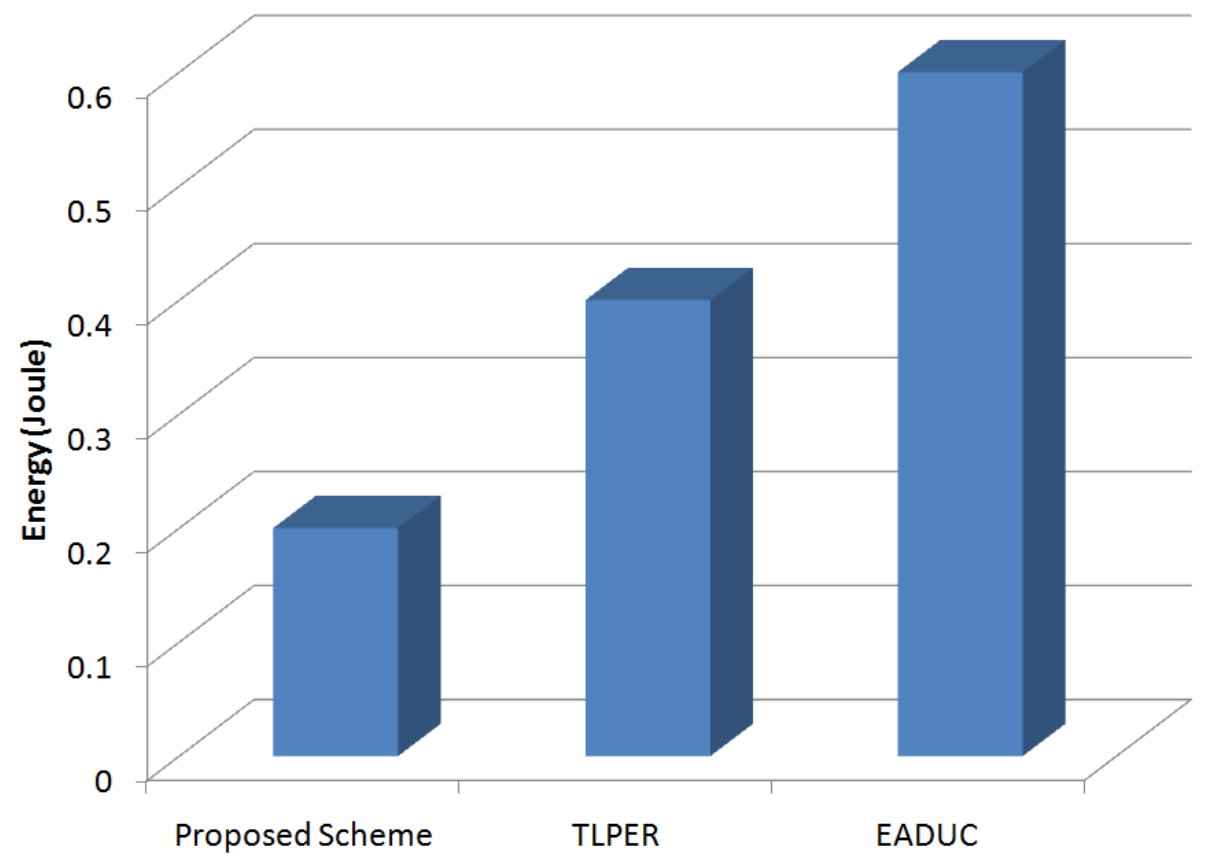

Fig.3. Energy Consumption in different methodologies and proposed methodology

\section{Conclusion}

In order to decrease the hop-by-hop communication, the large scale networks are divided in group of regions using clustering. If the clustering algorithm used proves to be inefficient, it will result in the consumption of more energy, thereby affecting the ndoes battery when delivering data packets. Further, in order to ensure less utilization of the battery resource while transferring data from source to destination within the minimum stipulated time, an appropriate routing algorithm is essential. To get maximum utilization of the network and to further enhance the network lifetime, routing and clustering techniques are combined. The proposed research work gives a design of routing and clustering. Routing in the proposed paper is dependent on intracluster and intercluster communication using node selection by forwarding in order to increase the network's throughput. The results show that the proposed work reduces hop-by-hop communication, minimizes formation of maximum clusters and requires less energy.

\section{References}

[1] Sarkar, A., \& Murugan, T. S. (2019). Cluster head selection for energy efficient and delay-less routing in wireless sensor network. Wireless Networks, 25(1), 303-320.

[2] Wang, J., Gao, Y., Liu, W., Sangaiah, A. K., \& Kim, H. J. (2019). An improved routing schema with special clustering using PSO algorithm for heterogeneous wireless sensor network. Sensors, 19(3), 671.

[3] Diwakaran, S., Perumal, B., \& Devi, K. V. (2019). A cluster prediction model-based data collection for energy efficient wireless sensor network. The Journal of Supercomputing, 75(6), 3302-3316. 
Journal of ISMAC (2020)

Vol.02/ No.03

Pages: $140-146$

http://irojournals.com/iroismac/

DOI: https://doi.org/10.36548/jismac.2020.3.001

[4] Robinson, Y. H., Julie, E. G., \& Kumar, R. (2019). Probability-based cluster head selection and fuzzy multipath routing for prolonging lifetime of wireless sensor networks. Peer-to-Peer Networking and Applications, 12(5), 1061-1075.

[5] Raj, J. S. (2019). QoS optimization of energy efficient routing in IoT wireless sensor networks. Journal of ISMAC, 1(01), 12-23.

[6] Shalini, V. B., \& Vasudevan, V. (2019). Achieving energy efficient wireless sensor network by choosing effective cluster head. Cluster Computing, 22(4), 7761-7768.

[7] Wan, Z., Liu, S., Ni, W., \& Xu, Z. (2019). An energy-efficient multi-level adaptive clustering routing algorithm for underwater wireless sensor networks. Cluster Computing, 22(6), 14651-14660.

[8] Yan, Z., Mukherjee, A., Yang, L., Routray, S., \& Palai, G. (2019). Energy-efficient node positioning in optical wireless sensor networks. Optik, 178, 461-466.

[9] Pacharaney, U. S., \& Gupta, R. K. (2019). Clustering and compressive data gathering in wireless sensor network. Wireless Personal Communications, 109(2), 1311-1331.

[10] Narendran, M., \& Prakasam, P. (2019). An energy aware competition based clustering for cluster head selection in wireless sensor network with mobility. Cluster Computing, 1-10.

[11] Yu J, Qi Y, Wang G, Guo Q and Gu X (2011) "an energy-aware distributed unequal clustering protocol for wireless sensor networks, " international journal of distributed sensor networks, vol. 2011

[12] RadiM, Dezfouli B, Bakar KA, LeeM(2012) Multipath routing in wireless sensor networks: survey and research challenges. Sensors 12(1):650-685.

[13] Raj, J. S. (2019). A comprehensive survey on the computational intelligence techniques and its applications. Journal of ISMAC, 1(03), 147-159.

ISSN: 2582-1369 (online)

Submitted: 16.05 .2020

Accepted: 29.06 .2020

Published: 06.07.2020 